\title{
A Neurofeedback Protocol for Executive Function to Reduce Depression and Rumination: A Controlled Study
}

\author{
Sheng-Hsiang Yu, Chao-Yuan Tseng, Wei-Lun Lin \\ Department of Psychology, Fo Guang University, Jiaosi, Yilan County, Taiwan
}

\begin{abstract}
Objective: Rumination is a maladaptive emotional-regulation strategy that is strongly associated with depression. Impaired executive function can lead to difficulties in disengaging from rumination, thus exacerbating depression. In this study, we inspect an electroencephalograph neurofeedback protocol that enhance the target peak alpha frequency (PAF) activation in the prefrontal region. We examine the protocol's effects on depression and rumination.

Methods: We randomly assigned 30 dysphoric participants into either the neurofeedback training group or the control group. We then evaluated their depression, rumination, and executive function at pre- and posttraining so as to examine the effects of the neurofeedback.

Results: The results show that this neurofeedback protocol can specifically enhance participants' target PAF. The participants' executive function performances significantly improved after undergoing 20 neurofeedback sessions. Compared with those in the control group, those in the neurofeedback group had significantly fewer depressive symptoms and significantly reduced rumination. Moreover, as target PAF and executive function improved, depression and rumination both declined.

Conclusion: Our data are in line with those of previous studies that indicated a relationship between upper-band alpha activity and executive function. This PAF neurofeedback can effectively enhance participants' executive function, which can reduce rumination and ameliorate depression. This neurofeedback training is based on basic cognitive neuroscience, so it sheds light on depression's pathological factors and etiology.
\end{abstract}

KEY WORDS: Depression; Electroencephalogram; Executive function; Neurofeedback; Rumination.

\section{INTRODUCTION}

Depressive disorder's high relapse rate and chronic course make it one of the leading causes of disabilities in social functioning [1]. Researchers have suggested that impairment in emotion regulation plays a pathological role in depression maintenance and relapse [2-4]. Rumination involves a focused attention on the symptoms of one's depression, its possible causes, and its consequences-

Received: September 17, 2019/ Revised: November 8, 2019

Accepted: March 21, 2020

Address for correspondence: Sheng-Hsiang Yu

Department of Psychology, Fo Guang University, No.160, Linwei

Rd., Jiaosi, Yilan County 26247, Taiwan

E-mail: yu.shenghsiang@gmail.com

ORCID: https://orcid.org/0000-0001-7173-3779

Wei-Lun Lin

Department of Psychology, Fo Guang University, No.160, Linwei

Rd., Jiaosi, Yilan County 26247, Taiwan

E-mail: wllin.fgu@gmail.com

ORCID: https://orcid.org/0000-0003-2477-0651 rather than its solutions [5]. Rumination is a maladaptive emotional-regulation strategy that is strongly associated with symptoms of various psychopathologies, including depression, anxiety, and eating disorders [6]. Individuals who tend to ruminate are more likely than other individuals to experience prolonged and worsening negative emotional states, as well as to subsequently develop depression $[4,5,7]$.

\section{Depression, Rumination, and Executive Function}

Recent theories and empirical results support the idea that emotion regulation - which involves cognitive functions such as monitoring, evaluating, and modifying emotional responses-relies on the executive function (EF) [8-10]. The literature demonstrates that major depressive disorder (MDD) is reliably associated with broad impairments in multiple cognitive domains, especially the EF $[11,12]$. Researchers have also indicated that the re-

(ㄷ) This is an Open-Access article distributed under the terms of the Creative Commons Attribution Non-Commercial License (http://creativecommons.org/licenses/by-nc/4.0) which permits unrestricted non-commercial use, distribution, and reproduction in any medium, provided the original work is properly cited. 
peated, difficult-to-control characteristics of ruminative responses are associated with executive dysfunction [13-15]. Individuals who tend to ruminate have difficulty disengaging from negative materials [16-18].

Neurophysiological evidence also indicates the presence of close links between EF and both depression and rumination $[19,20]$. In comparison with healthy adults, MDD patients have less activation in the prefrontal regions when processing emotional stimuli $[21,22]$. Rumination, which is characterized by frequent negative emotional responses and low cognitive control, is also related to functional abnormalities in the prefrontal brain [15, $19,23]$. Recent electroencephalographic (EEG) studies indicate that hypoactivation of the prefrontal cortex is associated with self-related rumination [24-26].

\section{Neuromodulatory Techniques for Depression and Executive Function}

Given its impact on normal functioning and its close relationship with depression, scholars have proposed many interventions to enhance EF and thus reduce depression symptoms; these include cognitive control training [27] and exercise training [28]. Neuromodulatory techniques, for example, repetitive transcranial magnetic stimulation, which induce changes in prefrontal activities, have been shown to ameliorate MDD patients' depression symptoms [29-31] and improve their cognitive functions [32,33]. Another noninvasive technique that can effectively modulate cortical activity and that could thus serve as an intervention against depression is EEG neurofeedback (NF) training [34,35]. EEG NF training can effectively activate the prefrontal lobe and thus enhance the brain's neural plasticity [36]. This technique is meant to enable individuals to learn to regulate their EEG activity through visual or acoustic feedback. Moreover, researchers have hypothesized that a change in EEG activity is associated with a change in cortical activation, thus reducing symptomatology $[37,38]$. Scholars have designed several EEG NF protocols to intervene in depression, including a protocol that helps depressed patients to increase beta activation and decrease theta activation in the frontal region [39] or to reduce frontal alpha asymmetry [40-42].

EEG NF can improve individuals' performances in several EF-related cognitive tasks [43]. Based on the association between inhibitory processes in working memory and upper alpha-band activity [44-47], NF protocols meant to enhance upper alpha activation have been shown to improve working memory performance [48-50] and mental rotation $[51,52]$. Higher ordered cognition performances involve the peak alpha frequency (PAF)an index of an individual's level of cognitive preparedness $[44,53,54]$. Angelakis and his colleges [55] indicated that PAF NF can improve cognitive processing speed and EF in older adults, whose average PAF is lower than that of young adults. Notably, researchers have recently found significant correlations between PAF and depression [56, 57]. Applying PAF NF training to MDD patients enhanced their working memory and EF performances [58,59]. However, the authors of these studies have not reported whether this NF protocol can reduce depression symptoms in MDD patients.

In sum, scholars have hypothesized that impaired EF is a crucial factor in ruminative responses and depression symptomatology. PAF NF has the potential to activate the prefrontal region and thus enhancing $\mathrm{EF}$ among depressed individuals. To our knowledge, however, few researchers have examined PAF NF's effects on cognitive function and on the behavioral level (in terms of rumination and depressive symptoms). Thus, in this study, we aimed to determine whether PAF NF can enhance EF and reduce dysphoric participants' rumination and depression symptoms.

\section{METHODS}

\section{Participants}

We first screened 300 students in Fo Guang University by the Chinese Response Style Questionnaire Short Form and the Chinese version of the beck depression inventory-II (BDI-II). We then recruited 30 participants who had symptoms of severe depression and high tendencies toward rumination (in the top third of the sample) to participate in the follow-up experiment.

We randomly assigned participants into the NF training group (who underwent the NF training for 20 sessions, 2 or 3 times per week) or the sham control (SC) group (who underwent brainwave measurement for 20 sessions, also 2 or 3 times per week, but with no feedback provided); each group had 15 participants. One participant in the NF group and three participants in SC group dropped out of the study due to personal issues (such as getting sick or dropping out of college). Thus, in the final sample, there 
Table 1. Demographic characteristics and changes in depression and rumination in experimental group and control group

\begin{tabular}{lcc}
\hline \multicolumn{1}{c}{ Variable } & $\mathrm{NF}(\mathrm{n}=14)$ & $\mathrm{SC}(\mathrm{n}=12)$ \\
\hline Sex $(\mathrm{M} / \mathrm{F})^{\mathrm{a}}$ & $6 / 8$ & $6 / 6$ \\
Age $^{\mathrm{b}}$ & $22.33 \pm 2.43$ & $23.05 \pm 3.25$ \\
Baseline PAF $^{\mathrm{c}}$ & $1.59 \pm 2.34$ & $1.98 \pm 2.24$ \\
Depression & & \\
$\quad$ Pretraining & $24.9 \pm 8.25$ & $21.0 \pm 6.27$ \\
Posttraining & $16.8 \pm 9.24$ & $17.5 \pm 8.25$ \\
$t$ & $-3.31^{* *}$ & -1.76 \\
Rumination & & \\
$\quad$ Pretraining & $28.8 \pm 5.25$ & $27.7 \pm 3.14$ \\
Posttraining & $24.5 \pm 4.88$ & $26.3 \pm 2.31$ \\
$t$ & $-4.77^{* * *}$ & -1.40 \\
Distraction & & \\
Pretraining & $25.6 \pm 5.65$ & $24.1 \pm 4.71$ \\
Posttraining & $26.3 \pm 3.36$ & $23.6 \pm 5.35$ \\
$t$ & 0.53 & -0.52 \\
\hline
\end{tabular}

Values are presented as number only or mean \pm standard deviation. $\mathrm{NF}$, neurofeedback; SC, sham control; PAF, peak alpha frequency. ${ }^{\mathrm{a}} \chi^{2}=0.13, p=0.72,{ }^{\mathrm{b}} t=0.65, p=0.52,{ }^{\mathrm{C}} \mathrm{Mann}-$ Whitney $U=83, p=$ 0.53. ${ }^{* *} p<0.01 ;{ }^{* * *} p<0.001$.

were 14 participants in the NF group and 12 participants in the SC group. The demographical data of the present sample are presented in Table 1. There are no significant group differences in sex and age between the NF group and the SC group.

All the participants were right-handed and had normal hearing. The participants all completed an informed consent form and received NT 2,500 USD in recompense for their time. This study was approved by the research ethic committee of National Taiwan University (No. NTU-REC 201505ES055).

\section{Self-Report Questionnaires}

\section{Chinese beck depression inventory-II}

We used the Chinese version of the BDI-II [60], which is a translation of the original beck depression inventory-II [61] - a questionnaire that assesses the severity of depression symptoms during the previous 2 weeks. The BDI-II is a 21 -item measure that uses a 4-point Likert scale $(0-3)$, and the total score is the total of the ratings for all items (range: $0-63$ ). The BDI-II has excellent reliability and validity in US samples [61]. Both its internal consistency $(\alpha=0.94$, split half $=0.91)$ and its construct validity were good in a Taiwanese psychiatric sample [60].

\section{Chinese response style questionnaire, short form}

The Chinese response style questionnaire, short form [62] is the short form of the Chinese version of the response style questionnaire [63]. This questionnaire has 20 items. The participants evaluated the frequency of their responses regarding when they felt depressed. The questionnaire evaluates two factors (rumination and distraction), and there are 10 items for each factor. The participants rated the items on a 4-point Likert scale ranging from 1 (fully disagree) to 4 (fully agree), with higher scores indicating higher rumination or distraction. Both the internal consistency ( $\alpha=0.91$ and 0.89 for rumination and distraction, respectively) and the construct validity were good in Taiwanese samples [62].

\section{Executive Function Task}

We adopted the computerized Modified Sternberg Task [17] to measure EF performance. In each trial, following a 500-milliseconds fixation, three positive words and three negative words (in red or blue) were presented as two lists that the participant sought to remember for 7.8 seconds. After that, a colored box (also in red or blue) was presented as a cue for 1 second. The colored box indicates which one of the lists (either blue or red) is central and that the other list becomes irrelevant. A probe word appeared in black within that box, and the participant had to decide whether the probe word belonged to the assigned color list (i.e., whether it was relevant). A flow chart for this task is presented in Figure 1.

By changing the probe, we were able to calculate the negative intrusion effect; words in the negative list that were not relevant to a given cue (i.e., when the correct response was no) had longer response times. A longer response time indicates a larger intrusion effect. For example, if the cue is a red box, then the positive words are relevant and the negative words are irrelevant. For a probe that shows a negative word (e.g., anger) in a red box, the no response is thus correct. Previous researchers of this task found that participants with MDD diagnoses had a greater negative intrusion effect than members of control groups; moreover, the intrusion effect was significantly associated with rumination [17]. In brief, a lower intrusion effect indicates a better ability to update working-memory contents, which in turn indicates better EF. 


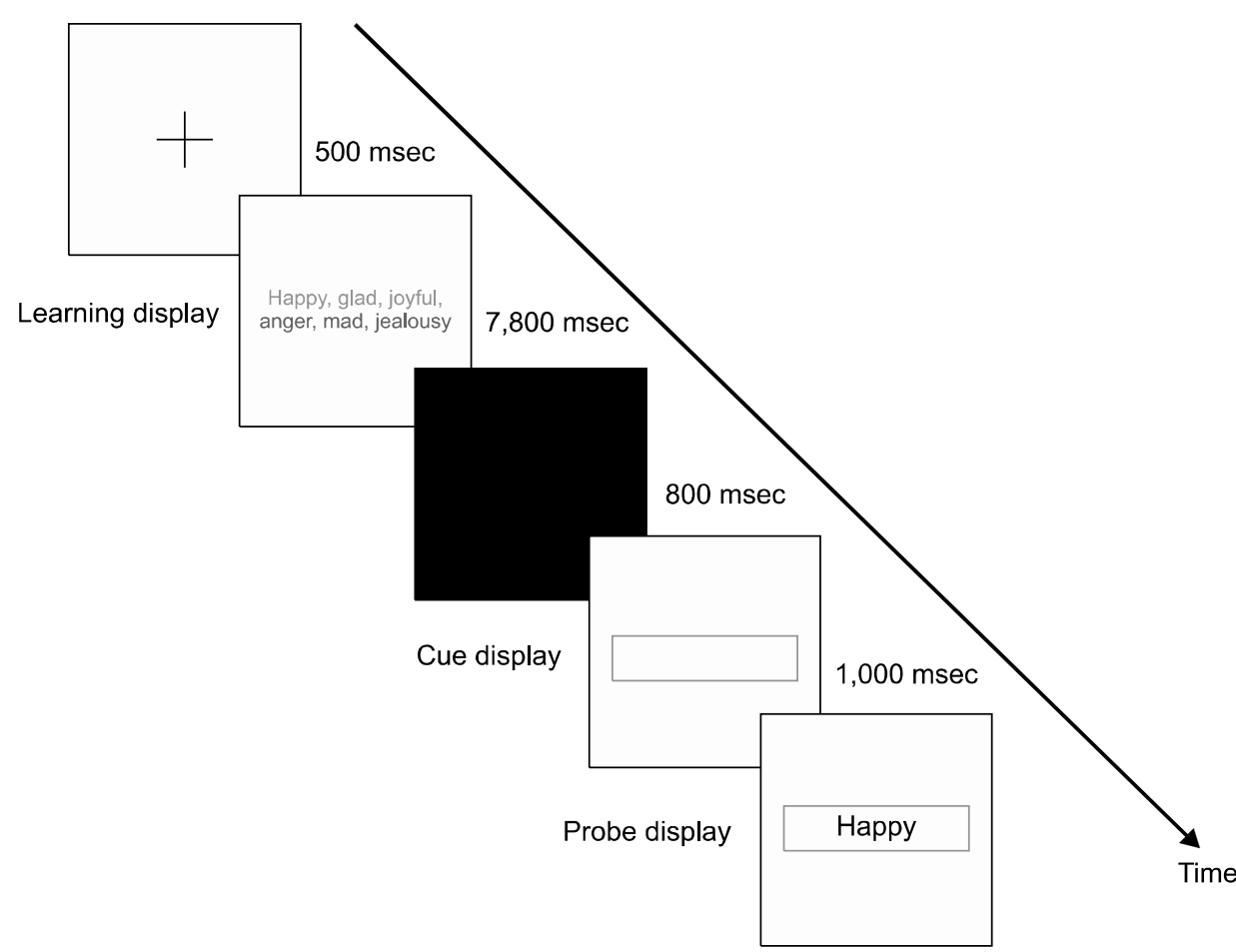

Fig. 1. The flowchart of Modified Sternberg Task.

\section{Neurofeedback Training}

In this study, NF was administered at prefrontal electrodes Fp1 and Fp2 in the eyes-open condition with ProComp Infiniti Biofeedback System (Thought Technology Ltd., Montreal, QC, Canada). The NF training focused on the enhancement of target PAF activation of prefrontal brain. According to Angelakis et al. [55], the PAF range is 10 to $11 \mathrm{~Hz}$ in young adults; this range is related to EF performances. Hence, we selected the activation range of 10 to $11 \mathrm{~Hz}$ as the target PAF band in this study. In order to evaluate the effectiveness of this newly developed NF protocol in the manner of group comparison, we adopted a standardized feedback for every participant in the NF group. The feedback threshold was set as a $10-11 \mathrm{~Hz}$ amplitude above $5 \mu \mathrm{V}$ that lasted at least 0.25 milliseconds. The number of $10-11 \mathrm{~Hz}$ activation, which was counted when the power of EEG activity was above the training threshold, represented estimated PAF magnitude in the training session. This operational definition is similar to that of Angelakis et al. [55]. It has the advantage to decrease the large variance of absolute brainwave power, and has shown its effectiveness and practicability in young adults in the previous study in other areas [64].

According to the International 10-20 System, we placed the monopolar EEG electrodes at Fp1 and Fp2, used a sampling rate of $2,048 \mathrm{~Hz}$, and placed the reference electrodes in the ipsilateral ear (A1 and A2). There exist some NF studies that utilized electrode sites Fp1 and/or Fp2 [34,39,65]. The literature indicate that Fp1 and Fp2 mainly reflect the activities of anterior prefrontal cortex, especially the Brodmann area 10 (BA10) [66,67]. This region is suggested to be involved in multiple-task coordination and updating-specific EF $[68,69]$, which we expected to be associated with the performances in the Modified Sternberg Task in this study.

We developed a modified NF training procedure based on that of Vernon et al. [70]. The participants received 20 sessions of NF training (30 minutes each) over 10 weeks. In each training session, participants were asked to sit in a relaxed manner with their eyes open to avoid falling asleep and refrain from unnecessary movements until the end of the training session. Bazanova [71] suggested that the amplitude of alpha band activity in eye open condition can be validly depicted. The participants then engaged in four 150-seconds trials in which they tried to make the auditory feedback (a sound of pure tone of 400 $\mathrm{Hz}$ ) appear in a continuous and stable manner. Instead of visual feedback, auditory feedback was adopted to prevent possible eye blink artifacts. 


\section{Procedure}

The procedure included three parts: the pretraining evaluation of depression symptoms, rumination, and EF; the training sessions (i.e., the PAF NF training for the NF group and the auditory signal-detecting task for the SC group); and the posttraining evaluation of depression symptoms, rumination, and EF.

The procedure is shown in Figure 2. The procedure in the SC group was the same as that of the NF group, except that the participants tried to detect the target signal (the 400-Hz signal tone).

\section{Statistical Analysis}

All statistical analyses were conducted using IBM SPSS 20.0. (IBM SPSS Statistics for Windows ver. 20.0, 2011; IBM Corp., Armonk, NY, USA). To investigate the validation of the present NF protocol, three criteria proposed by Zoefel et al. [52], i.e., trainability, independence, and interpretability, were examined accordingly. To examine trainability, we excluded the data from the first training session because we treated that as a practice session. We thus analyzed the participants' brainwave data in the second through 20th sessions. For each subject, we calculated the average number of $10-11 \mathrm{~Hz}$ activations in each training session. We also conducted linear regressions for each participant and used the slope across training sessions to represent the level of enhancement in target PAF activity, called PAF slope. To inspect independence, we examined whether NF training enhanced the trained PAF at 10 to $11 \mathrm{~Hz}$ without affecting other alpha bands. We thus calculated the number of activations that were not in the PAF alpha band (i.e., 7-10
$\mathrm{Hz}$ or $11-13 \mathrm{~Hz}$ ) for the training blocks in each session. To test the interpretability, we examined the changes in EF performance for the NF and SC groups, both pre- and posttraining. Moreover, correlations among PAF slope and changes in depression, rumination, and EF were conducted.

To determine whether dysphoric individuals' rumination and depressive symptoms could be alleviated through the NF training, the change in depression and rumination (the difference between the posttraining and pretraining scores) in the NF group and in the SC group were compared.

\section{RESULTS}

\section{Change in Electroencephalograph Readings}

In terms of trainability, we found significant positive correlation between average number of target PAF activations and the session number $(r=0.38, p<0.05)$ in the NF group. On the other hand, we found no significant correlation between target PAF activation and the session number in the SC group $(r=-0.09)$.

In terms of independence, we found no significant correlations between the non-target PAF activations and the session number in the NF group $(r=-0.02)$. This result indicates that the training did not affect the activities in the other alpha frequency bands. All these results support the effectiveness and specificity of this study's NF training procedure in enhancing PAF at 10 to $11 \mathrm{~Hz}$, which means that this protocol fulfills the criteria of trainability and independence [52].

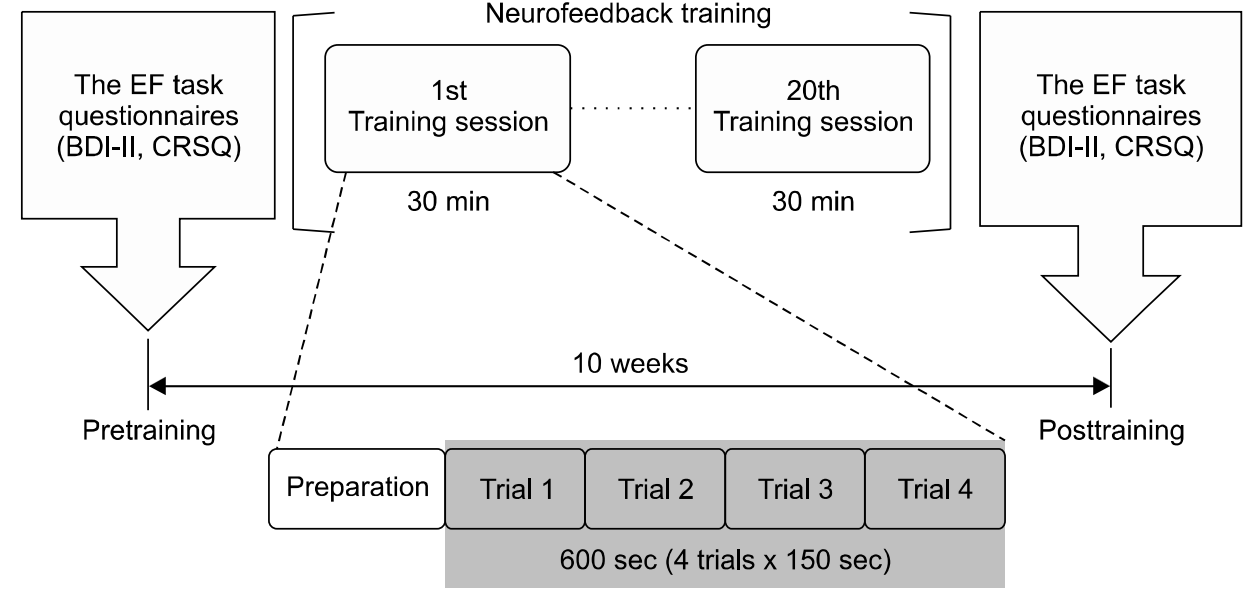

Fig. 2. The research procedure. $\mathrm{EF}$, executive function; $\mathrm{BDI}-\mathrm{II}$, beck depression inventory-II; CRSQ, Chinese Response Style Questionnaire. 


\section{Effects on Executive Function Performance}

We conducted Shapiro-Wilk tests to determine the normality of differences in EF performance (intrusion effect) and also in depression, rumination and distraction, which are described in the next paragraph. The results show that the data was all normal distributed ( $p=0.70,0.14,0.89$, and 0.12 , respectively). Figure 3 shows the changes in EF performance for the NF and SC groups, both pre- and posttraining. The results show that the NF group had a significant improvement in EF performance (i.e., a decreased intrusion effect) after training $(t=2.32, p<0.05)$; however, the control group showed no difference $(t=-1.85$, not significant [ns]). The EF-enhancing effect was significantly higher in the NF group (mean $[\mathrm{M}]=-263.50$, standard deviation $[\mathrm{SD}]=425.73)$ than in the SC group $(M=223.76, \mathrm{SD}=418.71), t(24)=-2.93, p<0.01$, with a large effect size (Cohen's $d=1.15$ ).

These results indicate that the NF group's participants significantly improved their ability to update content in their working memory after NF training. Thus, this protocol fulfills the criterion of interpretability.

\section{Effects on Depression and Rumination}

Table 1 presents the descriptive statistics and the difference between the groups. There were no group differ-

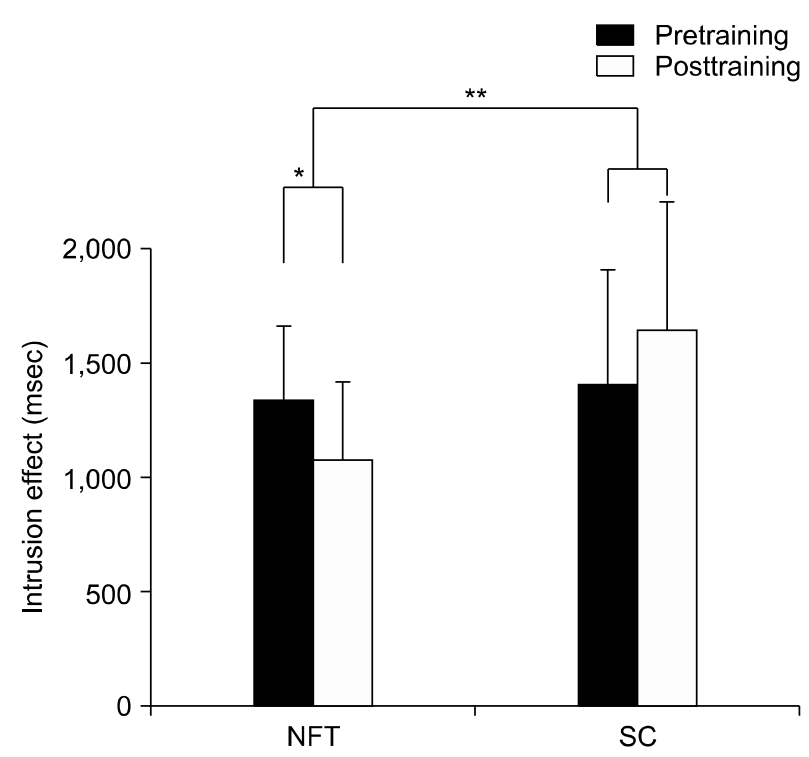

Fig. 3. The performances of EF in the pre- and posttraining evaluations.

$\mathrm{EF}$, executive function; SC, sham control; NFT, neurofeedback training.

${ }^{*} p<0.05,{ }^{* *} p<0.01$. ences in terms of sex, age, depression, rumination, or distraction in the pretraining evaluation (all $t \mathrm{~s}<1.31$, ns). The value of the baseline PAF was obtained by participants' target PAF activation in the trial 1 in the first training session. The nonparametric statistics was used because this variable was not normal distributed (ShapiroWilk $W=0.60, p<0.001)$. The result of nonparametric statistics test suggested that there is no significant difference in baseline PAF between groups (Table 1).

The results of the $t$ tests indicate that depression and rumination significantly decreased after training for the NF group but not for the SC group. Moreover, there were no significant changes in distraction in either the NF or SC groups. Between-group comparisons show that the change in depression (the difference between the posttraining and pretraining scores) was larger in the NF group than in the SC group, $t(24)=1.42$ (ns), with a medium effect size (Cohen's $d=0.56$ ). The change in rumination was significantly larger in the NF group than in the control group, $t(24)=2.13, p<0.05$, with a large effect size (Cohen's $d=$ 0.84).

As expected, these results show that NF training can relieve dysphoric college students' depression symptoms and reduce their rumination tendencies. Moreover, the results indicate a positive correlation between the change in EF performance and the changes in both depression ( $r=$ $0.38, p=0.18)$ and rumination $(r=0.30, p=0.29)$ for the NF group's participants. There were positive correlations among the PAF slope and changes in depression, rumination, and EF performance ( $r=0.42,0.26$, and 0.43 , respectively). The results suggest that the enhancement in target PAF activation through this NF protocol is related to the improvement in EF and to the reduction in rumination and depression. To sum up, these results show the protocol's effectiveness in terms of EF and indicate that greater improvements in EF correlate with greater reductions in depression and rumination.

\section{DISCUSSION}

Our aim in this study was to determine whether dysphoric individuals' cognitive deficits and depressive symptoms can be alleviated through NF training. Because prefrontal activation is associated with executive control $[22,72,73]$, we hypothesized that enhancing the prefrontal cortical activity would improve performance in 
higher order cognitive functions. Due to the exploratory nature of this study, we examined the validity of this protocol. The results satisfy the criteria that Zoefel et al. [52] suggested, i.e., trainability, independence, and interpretability. This NF protocol specifically enhanced the participants' target PAF activation $(10-11 \mathrm{~Hz})$ and did not alter the other alpha frequencies. In addition, the results support the hypothesis that this protocol enhances EF performance. More importantly, after the NF training, the dysphoric college students' depressive symptoms and cognitive vulnerability (rumination) were significantly reduced. Taken together, the present NF protocol has been demonstrated to be a valid and practical way to reduce rumination and ameliorate depression.

Our data are in line with those of previous studies that indicated a relationship between upper-band alpha activity and EF [48,50,52,55]. In Escolano et al. [58], NF training focused on the upper alpha band enhanced MDD patients' working memory performance but not their performance on the Stroop task. Researchers have recently indicated that alpha activation may play an active role in cortical inhibition rather than cortical idling $[74,75]$. Klimesch [76] suggested that alpha-band oscillations are closely linked to the suppression and selection of attention, which enables the control of knowledge access and semantic orientation. Segrave and his colleges [56] found that, during working-memory processing, patients with MDD displayed greater upper alpha-band event related synchronization than did control participants. Segrave et al. [56] also indicated that this phenomenon might be a neurobiological compensation strategy that allows depressed patients to maintain accurate performance in cognitive processing. The PAF (which we targeted in this protocol) could be associated with the processing of affective materials in the memory $[47,76,77]$. The Modified Sternberg Task used in the present study involves the ability to update materials in the working memory. Thus, this study provides preliminary evidence of the association between $\mathrm{PAF}$ and updating-specific EF, which relies on anterior prefrontal cortex (BA10) activity $[67,68]$.

According to the impaired disengagement hypothesis, impaired EF leads to difficulties in disengaging from negative, ruminative cognition, which further strengthens depressive symptoms [18,78-80]. In comparison with those who are able to update no-more-relevant negative thoughts effectively, individuals with poor updating EF are less like- ly to control their ruminative cognitions $[17,78,79]$. For individuals with high rumination tendency, brain activity increases when they try to change affective materials [81] and they need more resources than other individuals to discard negative self-related thoughts [23]. There is promising evidence that ruminators have stronger connectivity than other individuals between the default mode network's regions [82-84]. Some researchers indicated that depressive rumination is outcome of default mode network dysfunction, which may reflect poor executive control $[85,86]$. Some researchers suggested that spontaneous self-referential thought is accompanied by enhanced alpha activity in the default mode network $[25,87]$. Combining these results, the PAF activity related to $\mathrm{EF}$ may be associated with the processing of negative self-referential ruminations. Enhanced EF can provide more mental resources and can thus allow people to discard maladaptive thoughts or negative sematic memories, thus diminishing their rumination and depressive symptoms. Therefore, enhancing EF is a potential underlying mechanism to explain how PAF NF can reduce rumination and ameliorate depression.

The limitations of this study are as follow. First, the sample size was relatively small. Second, instead of adopting diagnosed depressive disorder population, the study recruited dysphoric college students who had milder depression severity as our participants. The generalization of the findings is yet to be examined. Third, the present NF protocol used a standardized feedback procedure. The threshold of feedback was not customized for each trainee. Last, the EF can be divided into various components, such as updating, inhibition, and shifting $[10,88]$. We only measured updating subcomponent EF in this study. The relationships between PAF and different EF subcomponents are still yet to be investigated. Further studies with larger samples and a wider variety of EF tasks are still needed. A study of the efficacy of this PAF NF protocol on individuals who have been diagnosed with MDD is also still needed. Moreover, an individualized training protocol with adjustable feedback threshold according to trainee's baseline and learning performance is worth developing $[89,90]$.

In conclusion, this study's results indicate that, in dysphoric participants, PAF NF training can enhance EF and thus alleviate depression and rumination. To our knowledge, this study is one of the first controlled studies on the 
effects that the PAF NF protocol has on EF and depressive symptoms. Our NF training design uses basic cognition and the cognitive neuroscience, so it sheds light on depression's pathological factors and etiology; it thus is an implication for interventions related to depressive disorders [35].

\section{Acknowledgments}

We would like to thank the anonymous reviewers for their careful reading of our manuscript and their many insightful comments and suggestions. This study is supported by Ministry of Science and Technology, Taiwan (MOST-106-2410-H-431-008-MY2 and MOST-104-2410H-431-006-).

\section{Conflicts of Interest}

No potential conflict of interest relevant to this article was reported.

\section{Author Contributions}

Conceptualization: Sheng-Hsiang Yu, Wei-Lun Lin. Data acquisition: Chao-Yuan Tseng. Formal analysis: Sheng-Hsiang Yu, Chao-Yuan Tseng. Funding: Sheng-Hsiang Yu. Writing - original draft: Sheng-Hsiang Yu. Writingreview \& editing: Sheng-Hsiang Yu, Wei-Lun Lin.

\section{ORCID}

Sheng-Hsiang Yu https://orcid.org/0000-0001-7173-3779

Chao-Yuan Tseng https://orcid.org/0000-0002-1689-1712

Wei-Lun Lin https://orcid.org/0000-0003-2477-0651

\section{REFERENCES}

1. Ferrari AJ, Charlson FJ, Norman RE, Patten SB, Freedman G, Murray CJ, et al. Burden of depressive disorders by country, sex, age, and year: findings from the global burden of disease study 2010. PLoS Med 2013;10:e1001547.

2. Abramson LY, Alloy LB, Hankin BL, Haeffel GJ, MacCoon DG, Gibb BE. Cognitive vulnerability-stress models of depression in a self-regulatory and psychobiological context. In: Gotlib IH, Hammen CL, editors. Handbook of depression. New York:Guilford Press;2002. p.268-294.

3. Gross JJ, Muñoz RF. Emotion regulation and mental health. Clin Psychol 1995;2:151-164.

4. Nolen-Hoeksema S. Further evidence for the role of psychosocial factors in depression chronicity. Clin Psychol 2000;7: 224-227.

5. Nolen-Hoeksema S, Wisco BE, Lyubomirsky S. Rethinking rumination. Perspect Psychol Sci 2008;3:400-424.
6. Aldao A, Nolen-Hoeksema S, Schweizer S. Emotion-regulation strategies across psychopathology: a meta-analytic review. Clin Psychol Rev 2010;30:217-237.

7. Alloy LB, Abramson LY, Safford SM, Gibb BE. The cognitive vulnerability to depression (CVD) project: current findings and future directions. In: Alloy $L B$, Riskind $J H$, editors. Cognitive vulnerability to emotional disorders. Mahwah: Lawrence Erlbaum Associates;2006. p.43-72.

8. Joormann J, Yoon KL, Siemer M. Cognition and emotion regulation. In: Kring AM, Sloan DM, editors. Emotion regulation and psychopathology: a transdiagnostic approach to etiology and treatment. New York:Guilford Press;2010. p.174-203.

9. Whitmer AJ, Gotlib IH. An attentional scope model of rumination. Psychol Bull 2013;139:1036-1061.

10. Miyake A, Friedman NP. The nature and organization of individual differences in executive functions: four general conclusions. Curr Dir Psychol Sci 2012;21:8-14.

11. Woo YS, Rosenblat JD, Kakar R, Bahk WM, Mclntyre RS. Cognitive deficits as a mediator of poor occupational function in remitted major depressive disorder patients. Clin Psychopharmacol Neurosci 2016;14:1-16.

12. Snyder HR. Major depressive disorder is associated with broad impairments on neuropsychological measures of executive function: a meta-analysis and review. Psychol Bull 2013;139:81-132.

13. Davis RN, Nolen-Hoeksema S. Cognitive inflexibility among ruminators and nonruminators. Cogn Ther Res 2000;24: 699-711.

14. Philippot P, Brutoux F. Induced rumination dampens executive processes in dysphoric young adults. J Behav Ther Exp Psychiatry 2008;39:219-227.

15. Snyder HR, Miyake A, Hankin BL. Advancing understanding of executive function impairments and psychopathology: bridging the gap between clinical and cognitive approaches. Front Psychol 2015;6:328.

16. De Lissnyder E, Koster EHW, Derakshan N, De Raedt R. The association between depressive symptoms and executive control impairments in response to emotional and non-emotional information. Cogn Emot 2010;24:264-280.

17. Joormann J, Gotlib IH. Updating the contents of working memory in depression: interference from irrelevant negative material. J Abnorm Psychol 2008;117:182-192.

18. Joormann J, Nee DE, Berman MG, Jonides J, Gotlib IH. Interference resolution in major depression. Cogn Affect Behav Neurosci 2010;10:21-33.

19. Auerbach RP, Webb CA, Gardiner CK, Pechtel P. Behavioral and neural mechanisms underlying cognitive vulnerability models of depression. J Psychother Integr 2013;23:222-235.

20. Krompinger JW, Simons RF. Cognitive inefficiency in depressive undergraduates: stroop processing and ERPs. Biol Psychol 2011;86:239-246.

21. Siegle GJ, Steinhauer SR, Thase ME, Stenger VA, Carter CS. 
Can't shake that feeling: event-related fMRI assessment of sustained amygdala activity in response to emotional information in depressed individuals. Biol Psychiatry 2002;51: 693-707.

22. Siegle GJ, Thompson W, Carter CS, Steinhauer SR, Thase ME. Increased amygdala and decreased dorsolateral prefrontal $B O L D$ responses in unipolar depression: related and independent features. Biol Psychiatry 2007;61:198-209.

23. Vanderhasselt MA, Kühn S, De Raedt R. Healthy brooders employ more attentional resources when disengaging from the negative: an event-related fMRI study. Cogn Affect Behav Neurosci 2011;11:207-216.

24. Ferdek MA, van Rijn CM, Wyczesany M. Depressive rumination and the emotional control circuit: an EEG localization and effective connectivity study. Cogn Affect Behav Neurosci 2016;16:1099-1113.

25. Knyazev GG, Savostyanov AN, Bocharov AV, Brak IV, Osipov EA, Filimonova EA, et al. Task-positive and task-negative networks in major depressive disorder: a combined fMRI and EEG study. J Affect Disord 2018;235:211-219.

26. Putnam KM, McSweeney LB. Depressive symptoms and baseline prefrontal EEG alpha activity: a study utilizing ecological momentary assessment. Biol Psychol 2008;77:237-240.

27. Siegle GJ, Ghinassi F, Thase ME. Neurobehavioral therapies in the 21st century: summary of an emerging field and an extended example of cognitive control training for depression. Cogn Ther Res 2007;31:235-262.

28. Nouchi R, Kawashima R. Improving cognitive function from children to old age: a systematic review of recent smart ageing intervention studies. Adv Neurosci 2014;2014:235479.

29. Möbius M, Lacomblé L, Meyer T, Schutter DJLG, Gielkens T, Becker ES, et al. Repetitive transcranial magnetic stimulation modulates the impact of a negative mood induction. Soc Cogn Affect Neurosci 2017; 12:526-533.

30. Lee S, Jang KI, Yoon S, Chae JH. The efficacy of miniaturized repetitive transcranial magnetic stimulation in patients with depression. Clin Psychopharmacol Neurosci 2019;17:409414.

31. Kar SK. Predictors of response to repetitive transcranial magnetic stimulation in depression: a review of recent updates. Clin Psychopharmacol Neurosci 2019;17:25-33.

32. Demirtas-Tatlidede A, Vahabzadeh-Hagh AM, Pascual-Leone A. Can noninvasive brain stimulation enhance cognition in neuropsychiatric disorders? Neuropharmacology 2013;64: 566-578.

33. Vanderhasselt MA, De Raedt R, Leyman L, Baeken C. Acute effects of repetitive transcranial magnetic stimulation on attentional control are related to antidepressant outcomes. J Psychiatry Neurosci 2009;34:119-126.

34. Hammond DC. Neurofeedback treatment of depression and anxiety. J Adult Dev 2005;12:131-137.

35. Linden DE. Neurofeedback and networks of depression. Dialogues Clin Neurosci 2014;16:103-112.
36. Enriquez-Geppert S, Huster RJ, Herrmann CS. Boosting brain functions: improving executive functions with behavioral training, neurostimulation, and neurofeedback. Int J Psychophysiol 2013;88:1-16.

37. Hammond DC. What is neurofeedback: an update. J Neurother 2007; 10:25-36.

38. Schoenberg PL, David AS. Biofeedback for psychiatric disorders: a systematic review. Appl Psychophysiol Biofeedback 2014;39:109-135.

39. Walker JE, Lawson R. FPO2 beta training for drug-resistant depression - a new protocol that usually reduces depression and keeps it reduced. J Neurother 2013;17:198-200.

40. Choi SW, Chi SE, Chung SY, Kim JW, Ahn CY, Kim HT. Is alpha wave neurofeedback effective with randomized clinical trials in depression? A pilot study. Neuropsychobiology 2011; 63:43-51.

41. Peeters F, Oehlen M, Ronner J, van Os J, Lousberg R. Neurofeedback as a treatment for major depressive disorder--a pilot study. PLoS One 2014;9:e91837.

42. Wang SY, Lin IM, Fan SY, Tsai YC, Yen CF, Yeh YC, et al. The effects of alpha asymmetry and high-beta down-training neurofeedback for patients with the major depressive disorder and anxiety symptoms. J Affect Disord 2019;257:287-296.

43. Gruzelier JH. EEG-neurofeedback for optimising performance. I: a review of cognitive and affective outcome in healthy participants. Neurosci Biobehav Rev 2014;44:124141.

44. Bazanova OM, Vernon D. Interpreting EEG alpha activity. Neurosci Biobehav Rev 2014;44:94-110.

45. Hummel F, Andres F, Altenmüller E, Dichgans J, Gerloff C. Inhibitory control of acquired motor programmes in the human brain. Brain 2002;125(Pt 2):404-420.

46. Klimesch W, Sauseng P, Hanslmayr S. EEG alpha oscillations: the inhibition-timing hypothesis. Brain Res Rev 2007;53: 63-88.

47. Sauseng P, Klimesch W, Schabus M, Doppelmayr M. Frontoparietal EEG coherence in theta and upper alpha reflect central executive functions of working memory. Int I Psychophysiol 2005; 57:97-103.

48. Escolano C, Aguilar Herrero M, Minguez J. EEG-based upper alpha neurofeedback training improves working memory performance. In: 2011 Annual International Conference of the IEEE Engineering in Medicine and Biology Society; August 30-September 3, 2011; Boston. Abstract NR ThA03.6:23272330.

49. Escolano C, Oliván Blázquez B, López del Hoyo Y, García Campayo J, Minguez J. Double-blind single-session neurofeedback training in upper-alpha for cognitive enhancement of healthy subjects. In: 2012 Annual International Conference of the IEEE Engineering in Medicine and Biology Society; August 28-September 1, 2012; San Diego. Abstract NR FrB16.12:4643-4647.

50. Nan W, Rodrigues JP, Ma J, Qu X, Wan F, Mak PI, et al. 
Individual alpha neurofeedback training effect on short term memory. Int J Psychophysiol 2012;86:83-87.

51. Hanslmayr S, Sauseng P, Doppelmayr M, Schabus M, Klimesch W. Increasing individual upper alpha power by neurofeedback improves cognitive performance in human subjects. Appl Psychophysiol Biofeedback 2005;30:1-10.

52. Zoefel B, Huster RJ, Herrmann CS. Neurofeedback training of the upper alpha frequency band in EEG improves cognitive performance. Neuroimage 2011;54:1427-1431.

53. Angelakis E, Lubar JF, Stathopoulou S. Electroencephalographic peak alpha frequency correlates of cognitive traits. Neurosci Lett 2004;371:60-63.

54. Angelakis E, Lubar JF, Stathopoulou S, Kounios J. Peak alpha frequency: an electroencephalographic measure of cognitive preparedness. Clin Neurophysiol 2004; 115:887-897.

55. Angelakis E, Stathopoulou S, Frymiare JL, Green DL, Lubar JF, Kounios J. EEG neurofeedback: a brief overview and an example of peak alpha frequency training for cognitive enhancement in the elderly. Clin Neuropsychol 2007;21:110-129.

56. Segrave RA, Thomson RH, Cooper NR, Croft RJ, Sheppard DM, Fitzgerald PB. Upper alpha activity during working memory processing reflects abnormal inhibition in major depression. J Affect Disord 2010;127:191-198.

57. Tement S, Pahor A, Jaušovec N. EEG alpha frequency correlates of burnout and depression: the role of gender. Biol Psychol 2016;114:1-12.

58. Escolano C, Navarro-Gil M, Garcia-Campayo J, Congedo M, De Ridder D, Minguez J. A controlled study on the cognitive effect of alpha neurofeedback training in patients with major depressive disorder. Front Behav Neurosci 2014;8:296.

59. Escolano C, Navarro-Gil M, Garcia-Campayo J, Minguez J. The effects of a single session of upper alpha neurofeedback for cognitive enhancement: a sham-controlled study. Appl Psychophysiol Biofeedback 2014;39:227-236.

60. Lu ML, Che HH, Chang SW, Shen WW. Reliability and validity of the beck depression inventory-II. Taiwan J Psychiatry 2002;16:301-310.

61. Beck AT, Steer RA, Brown GK. BDI-II, Beck depression inventory: manual. 2nd ed. San Antonio:Psychological Corporation; 1996.

62. $\mathrm{Yu} \mathrm{SH}$, Chen $\mathrm{SH}$, Chang KJ. Rumination predicting depression and PTSD symptoms in postoperative breast cancer patients. Chin J Psychol 2008;50:289-302.

63. Nolen-Hoeksema S, Morrow J. A prospective study of depression and posttraumatic stress symptoms after a natural disaster: the 1989 Loma Prieta Earthquake. J Pers Soc Psychol 1991;61:115-121.

64. Lin WL, Shih YL. Designing EEG neurofeedback procedures to enhance open-ended versus closed-ended creative potentials. Creat Res J 2016;28:458-466.

65. Jeong $\mathrm{E}$, Lee J, Jung $\mathrm{H}$, Youn MK. The effect of the integrated therapy of neurofeedback, brain gymnastics, and oriental herbal tea on the improvement of brain functions and the quality of life of elders living alone. J Korea Acad Ind Coop Soc 2016;17:569-581.

66. Koessler L, Maillard L, Benhadid A, Vignal JP, Felblinger J, Vespignani $\mathrm{H}$, et al. Automated cortical projection of EEG sensors: anatomical correlation via the international 10-10 system. Neuroimage 2009;46:64-72.

67. Homan RW, Herman J, Purdy P. Cerebral location of international 10-20 system electrode placement. Electroencephalogr Clin Neurophysiol 1987;66:376-382.

68. Reineberg AE, Andrews-Hanna JR, Depue BE, Friedman NP, Banich MT. Resting-state networks predict individual differences in common and specific aspects of executive function. Neuroimage 2015;104:69-78.

69. Gilbert SJ, Spengler S, Simons JS, Steele JD, Lawrie SM, Frith $\mathrm{CD}$, et al. Functional specialization within rostral prefrontal cortex (area 10): a meta-analysis. J Cogn Neurosci 2006;18: 932-948.

70. Vernon D, Egner T, Cooper N, Compton T, Neilands C, Sheri $\mathrm{A}$, et al. The effect of training distinct neurofeedback protocols on aspects of cognitive performance. Int J Psychophysiol 2003:47:75-85.

71. Bazanova O. Comments for current interpretation EEG alpha activity: a review and analysis. J Behav Brain Sci 2012;2: 239-248.

72. Alvarez JA, Emory E. Executive function and the frontal lobes: a meta-analytic review. Neuropsychol Rev 2006;16:17-42.

73. Hunter SJ, Edidin JP, Hinkle CD. The developmental neuropsychology of executive functions. In: Hunter SJ, Sparrow EP, editors. Executive function and dysfunction: identification, assessment and treatment. Cambridge:Cambridge University Press;2012. p.17-36.

74. Cooper NR, Croft RJ, Dominey SJ, Burgess AP, Gruzelier JH. Paradox lost? Exploring the role of alpha oscillations during externally vs. internally directed attention and the implications for idling and inhibition hypotheses. Int I Psychophysiol 2003;47:65-74.

75. Jensen O, Mazaheri A. Shaping functional architecture by oscillatory alpha activity: gating by inhibition. Front Hum Neurosci 2010:4:186.

76. Klimesch W. $\alpha$-band oscillations, attention, and controlled access to stored information. Trends Cogn Sci 2012;16: 606-617.

77. Klimesch W. EEG alpha and theta oscillations reflect cognitive and memory performance: a review and analysis. Brain Res Brain Res Rev 1999;29:169-195.

78. De Raedt R, Koster EH. Understanding vulnerability for depression from a cognitive neuroscience perspective: a reappraisal of attentional factors and a new conceptual framework. Cogn Affect Behav Neurosci 2010;10:50-70.

79. Koster EH, De Lissnyder E, Derakshan N, De Raedt R. Understanding depressive rumination from a cognitive science perspective: the impaired disengagement hypothesis. Clin Psychol Rev 2011;31:138-145. 
80. Levens SM, Gotlib IH. Updating positive and negative stimuli in working memory in depression. J Exp Psychol Gen 2010; 139:654-664.

81. Chuen Yee Lo B, Lau S, Cheung SH, Allen NB. The impact of rumination on internal attention switching. Cogn Emot 2012; 26:209-223.

82. Berman MG, Misic B, Buschkuehl M, Kross E, Deldin PJ, Peltier S, et al. Does resting-state connectivity reflect depressive rumination? A tale of two analyses. Neuroimage 2014; 103:267-279.

83. Kaiser RH, Andrews-Hanna JR, Wager TD, Pizzagalli DA. Large-scale network dysfunction in major depressive disorder: a meta-analysis of resting-state functional connectivity. JAMA Psychiatry 2015;72:603-611.

84. Piguet C, Desseilles M, Sterpenich V, Cojan Y, Bertschy G, Vuilleumier $\mathrm{P}$. Neural substrates of rumination tendency in non-depressed individuals. Biol Psychol 2014;103:195-202.

85. Fox KC, Spreng RN, Ellamil M, Andrews-Hanna JR, Christoff $\mathrm{K}$. The wandering brain: meta-analysis of functional neuroimaging studies of mind-wandering and related spontaneous thought processes. Neuroimage 2015;111:611-621.

86. Konjedi S, Maleeh R. A closer look at the relationship between the default network, mind wandering, negative mood, and depression. Cogn Affect Behav Neurosci 2017;17:697-711.

87. Knyazev GG, Savostyanov AN, Volf NV, Liou M, Bocharov AV. EEG correlates of spontaneous self-referential thoughts: a cross-cultural study. Int J Psychophysiol 2012;86:173-181.

88. Miyake A, Friedman NP, Emerson MJ, Witzki AH, Howerter A, Wager TD. The unity and diversity of executive functions and their contributions to complex "Frontal Lobe" tasks: a latent variable analysis. Cogn Psychol 2000;41:49-100.

89. Enriquez-Geppert S, Huster RJ, Herrmann CS. EEG-neurofeedback as a tool to modulate cognition and behavior: a review tutorial. Front Hum Neurosci 2017;11:51.

90. Bioulac S, Purper-Ouakil D, Ros T, Blasco-Fontecilla H, Prats M, Mayaud L, et al. Personalized at-home neurofeedback compared with long-acting methylphenidate in an European non-inferiority randomized trial in children with $A D H D$. BMC Psychiatry 2019;19:237. 